\title{
Diffusible signal factor (DSF) quorum sensing signal and structurally related molecules enhance the antimicrobial efficacy of antibiotics against some bacterial pathogens
}

\author{
Yinyue Deng ${ }^{1,2}$, Amy Lim¹, Jasmine Lee ${ }^{1}$, Shaohua Chen², Shuwen An ${ }^{1}$, Yi-Hu Dong ${ }^{1}$ and Lian-Hui Zhang ${ }^{1,2^{*}}$
}

\begin{abstract}
Background: Extensive use of antibiotics has fostered the emergence of superbugs that are resistant to multidrugs, which becomes a great healthcare and public concern. Previous studies showed that quorum sensing signal DSF (diffusible signal factor) not only modulates bacterial antibiotic resistance through intraspecies signaling, but also affects bacterial antibiotic tolerance through interspecies communication. These findings motivate us to exploit the possibility of using DSF and its structurally related molecules as adjuvants to influence antibiotic susceptibility of bacterial pathogens.

Results: In this study, we have demonstrated that DSF signal and its structurally related molecules could be used to induce bacterial antibiotic susceptibility. Exogenous addition of DSF signal (cis-11-methyl-2-dodecenoic acid) and its structural analogues could significantly increase the antibiotic susceptibility of Bacillus cereus, possibly through reducing drug-resistant activity, biofilm formation and bacterial fitness. The synergistic effect of DSF and its structurally related molecules with antibiotics on $B$. cereus is dosage-dependent. Combination of DSF with gentamicin showed an obviously synergistic effect on B. cereus pathogenicity in an in vitro model. We also found that DSF could increase the antibiotic susceptibility of other bacterial species, including Bacillus thuringiensis, Staphylococcus aureus, Mycobacterium smegmatis, Neisseria subflava and Pseudomonas aeruginosa.
\end{abstract}

Conclusion: The results indicate a promising potential of using DSF and its structurally related molecules as novel adjuvants to conventional antibiotics for treatment of infectious diseases caused by bacterial pathogens.

\section{Background}

Antibiotics, which act by either killing or stopping microbial growth, have been used extensively in the control and prevention of infectious diseases. However, this live-or-die selection pressure has inevitably fostered the emergence of superbugs which are resistant to a range of conventional antibiotics. Infections associated with antibiotic-resistant pathogens are becoming more and more common in clinical and nosocomial settings [1,2], which become severe healthcare and public concerns.

\footnotetext{
* Correspondence: lianhui@imcb.a-star.edu.sg

${ }^{1}$ Institute of Molecular and Cell Biology, Proteos, 61 Biopolis Drive,

Singapore 138673, Singapore

${ }^{2}$ Guangdong Province Key Laboratory of Microbial Signals and Disease Control, College of Natural Resources and Environment, South China Agricultural University, Guangzhou, People's Republic of China
}

In addition, antibiotics are commonly associated with a range of adverse effects [3]. For instance, treatment using aminoglycoside antibiotics, such as gentamicin and kanamycin, can cause serious side effects, including balance difficulty, hearing loss, and nephrotoxicity $[4,5]$. Reduction and limitation of antibiotic usage is therefore of critical importance in clinical treatment of microbial infections.

Combination antibiotics containing more than one antimicrobial agent are designed to either improve efficacy through synergistic action of the agents, or overcome the bacterial resistance. This method has been effectively used for treatment of tuberculosis, leprosy, malaria, HIV, infections associated with cystic fibrosis, and infective endocarditis [6-9]. Currently, antibiotic combinations are frequently used to provide empirical

\section{() Biomed Central}


treatment for serious infections. However, given the facts that effective antibiotic combinations are still limited and superbugs are emerging rapidly, it is essential to continue to search for effective antibiotic combinations and other novel approaches to control infectious diseases. Recently, using nonantibiotic molecules to enhance the antibacterial efficacy of antibiotics offers a new kind of opportunity to practice a previously untapped expanse of clinical treatments. A few combinations of nonantibiotics with antibiotics showed increased activity against bacterial pathogens in vitro and in vivo [8,10-12].

The diffusible signal factor (DSF), which was originally found in Xanthomonas campestris pv campestris (Xcc), represents a new family of widely conserved quorum sensing (QS) signals in many Gram-negative bacterial species. It has been well-established that DSF-family signals play important roles in regulation of various biological functions such as biofilm formation, motility, virulence and antibiotic resistance [13-21]. In addition to their key roles in intraspecies signaling, the importance of DSF-family signals in interspecies and inter-kingdom communication has also been recognized [18,22]. It was reported that DSF signals from Burkholderia cenocepacia and Stenotrophomonas maltophilia modulate the virulence, antibiotic resistance and persistence of Pseudomonas aeruginosa in the cystic fibrosis airway [23,24]. Furthermore, it was found that an DSF-family signal produced by $P$. aeruginosa not only disperses its own biofilm formation but could also induce dispersion of biofilms of Escherichia coli, Klebsiella pneumoniae, Proteus mirabilis, Streptococcus pyogenes, Bacillus subtilis, Staphylococcus aureus, and the yeast Candida albicans [25]. Moreover, DSF-family signals showed a high level of potency in interference of the morphology transition of $C$. albicans $[14,17,22]$, which is a critical feature associated with the virulence of this pathogen.

Given the fact that biofilm formation is related to antibiotic resistance [26], together with the role of DSFfamily signals in regulation of bacterial biofilm formation and antibiotic resistance, we speculate that DSF-family signals may have a role in modulation of bacterial antibiotic susceptibility. In this study, we report that in the presence of DSF signal and its derivatives, some of which were identified as bacterial quorum sensing (QS) signals $[13,14,18,22]$, the minimum inhibitory concentrations (MIC) of a few antibiotics against the bacterial pathogens were significantly reduced. Furthermore, we showed that supplementation of DSF signal could substantially enhance the antimicrobial activity of gentamicin and reduce the cytotoxicity of $B$. cereus in an in vitro infection model. Our findings suggest the promising potentials of DSF and its structurally related molecules as putative antibiotic adjuvants for the control of bacterial infections.

\section{Results}

DSF and its structurally related molecules increase the antibiotic susceptibility of $B$. cereus

Bacillus is a genus of Gram-positive, rod-shaped bacteria. They are ubiquitous in nature, and consisting of both free-living and pathogenic species. Bacillus bacteria produce oval endospores to endure a wide range of extreme environmental conditions, while keeping the capacity to return to vegetative growth [27]. This remarkable characteristics of the endospore-vegetative cell transition of Bacillus pathogens allows them to be utilized as biological weapons $[28,29]$. Interestingly, our preliminary results showed that this morphological transition between the vegetative cell and endospore of Bacillus species could be stopped by exogenous addition of DSF-family signals (Deng, unpublished data). This finding, together with the previous observations that DSF signals are involved in regulation of bacterial biofilm formation, antibiotic tolerance and fungal morphological transition [15,22-24], we speculated that DSF-family signals may affect the bacterial antibiotic sensitivity of Bacillus cells. To test this hypothesis, we firstly chose B. cereus, which is a common human pathogen and causes foodborne illness such as nausea, vomiting and diarrhea [30], to assay the antibiotic susceptibility in the presence of DSF signal or its derivatives (Table 1). The result showed that except for T8-DSF, T15DSF and C8-DSF, all the other DSF-family signals and structurally related molecules displayed significantly synergistic effects with gentamicin (Figure 1A), which is an aminoglycoside and inhibits bacterial protein synthesis mainly through binding with the $30 \mathrm{~S}$ ribosomal subunit. In particular, addition of T14-DSF or C15-DSF decreased the MIC of gentamicin against B. cereus from $8.0 \mu \mathrm{g} / \mathrm{ml}$ to $0.0625 \mu \mathrm{g} / \mathrm{ml}$, which represents a 128 -fold difference (Figure 1A). Similarly, addition of DSF and related molecules to $B$. cereus culture also enhanced the bacterial susceptibility to kanamycin from 2- to 64-fold with T14DSF showing the strongest synergistic activity (Figure 1B). Interestingly, kanamycin is also an aminoglycoside that interacts with the $30 \mathrm{~S}$ subunit of prokaryotic ribosomes and inhibits protein synthesis. Compared to the strong synergistic effect on gentamicin and kanamycin, DSF and related molecules showed only moderate effects on rifampicin, addition of these molecules increased the antibiotic sensitivity of B. cereus up to 4-fold (Figure 1C). Different from gentamicin and kanamycin, rifampicin inhibits the DNA-dependent RNA polymerase in bacterial cells, thus preventing gene transcription to generate RNA molecules and subsequent translation to synthesize proteins.

The synergistic activity of DSF and its structurally related molecules with antibiotics on $B$. cereus is dosage-dependent To determine whether the synergistic activity of DSF with antibiotics is related to its dosages, DSF was 
Table 1 Chemical structure of DSF signal and its derivatives used in this study

\begin{tabular}{|c|c|c|c|}
\hline Compound & Configuration & Structure & References \\
\hline T8-DSF & trans & & 14 \\
\hline T10-DSF & trans & & 14 \\
\hline T11-DSF & trans & & 14 \\
\hline T12-DSF & trans & & 14 \\
\hline T13-DSF & trans & & 14 \\
\hline T14-DSF & trans & & 14 \\
\hline T15-DSF & trans & & 14 \\
\hline C8-DSF & cis & & 14 \\
\hline C10-DSF & cis & & 14 \\
\hline C11-DSF & cis & & 14 \\
\hline C12-DSF & cis & & 22 \\
\hline DSF & cis & & 14 \\
\hline C13-DSF & cis & & This study \\
\hline C14-DSF & cis & & 14 \\
\hline C15-DSF & cis & & 14 \\
\hline S12-DSF & NT & & This study \\
\hline
\end{tabular}

supplemented to the growth medium at various final concentrations, and MICs of gentamicin and kanamycin against $B$. cereus were tested. The results showed that activity of DSF signal on $B$. cereus sensitivity to gentamicin and kanamycin was dependent on the final concentration of the signal molecule (Figure 2A). Addition of DSF at a final concentration from $5-50 \mu \mathrm{M}$ increased the antibiotic susceptibility of $B$. cereus to gentamicin by 2 - to 16-fold, respectively (Figure 2A). Similarly, as shown in Figure 2A, combination of different final concentrations of DSF signal with kanamycin increased the synergistic activity by 1.3 - to 16 -fold.

To test the dosage-dependent synergistic activity of other DSF related molecules, we selected C13-DSF, which was prepared abundantly in our laboratory, as a representative molecule for further analysis. As shown in Figure 2B, the effects of C13-DSF on $B$. cereus sensitivity to gentamicin and kanamycin were also dosage-dependent. Addition of C13-DSF at a final concentration from $10 \mu \mathrm{M}$ to $50 \mu \mathrm{M}$ increased the gentamicin susceptibility of $B$. cereus by 2 - to 32 -fold, and similarly, increased the bacterial kanamycin susceptibility by about 2 - to 16 -fold (Figure 2B).

Combination of DSF signal with gentamicin synergistically decreases $B$. cereus pathogenicity in in vitro assays

We then continued to investigate the possibility of using DSF signal as antibiotics adjuvant for the therapy of infectious diseases caused by bacterial pathogens. HeLa cells were used as the in vitro model to test the synergistic activity of DSF signal with antibiotics against $B$. cereus. Results showed that exogenous addition of gentamycin significantly decreased the cytotoxicity of $B$. cereus to HeLa cell. For $2.5 \mathrm{~h}$ inoculation, the cytotoxicity of B. cereus was reduced by $11.15 \%, 17.95 \%$, and $26.9 \% \%$ with supplementation of 2,4 , and $8 \mu \mathrm{g} / \mathrm{ml}$ gentamycin, respectively (Figure 3 ). In contrast, combination of $50 \mu \mathrm{M}$ DSF signal with gentamycin led to more decreased cytotoxicity of B. cereus to HeLa cell than addition of the antibiotic alone. As shown in Figure 3, the cytotoxicity of B. cereus to HeLa cells was reduced by $26.9 \%, 29.15 \%$ and 36.4 with treatment of 2,4 , and $8 \mu \mathrm{g} / \mathrm{ml}$ gentamycin in combination with $50 \mu \mathrm{M}$ DSF, respectively. As a control, we found that DSF signal showed no cytotoxicity to HeLa cells and didn't affect the $B$. cereus virulence (Figure 3). These results not only further confirm the synergistic effect of DSF signal with antibiotics on B. cereus, but also highlight the potentials of using DSF and its structurally related molecules as adjuvants to antibiotics for treatment of infectious diseases caused by bacterial pathogens.

\section{DSF signal interferes with the drug-resistant activity, biofilm formation and persistence of $B$. cereus}

To elucidate the mode of action of DSF-family signals on $B$. cereus, we firstly analyzed the global gene expression patterns of B. cereus 10987 in the presence of DSF signal using microarray assay. It was revealed that addition of DSF signal significantly decreased the transcripts levels of the genes encoding a series of drug efflux systems and drug resistance proteinsof $B$. cereus (Additional file 1: Figure S1, Additional file 1: Table S1), which may likely reduce the antibiotic-resistant activity. We then tested the effect of DSF signal on B. cereus 


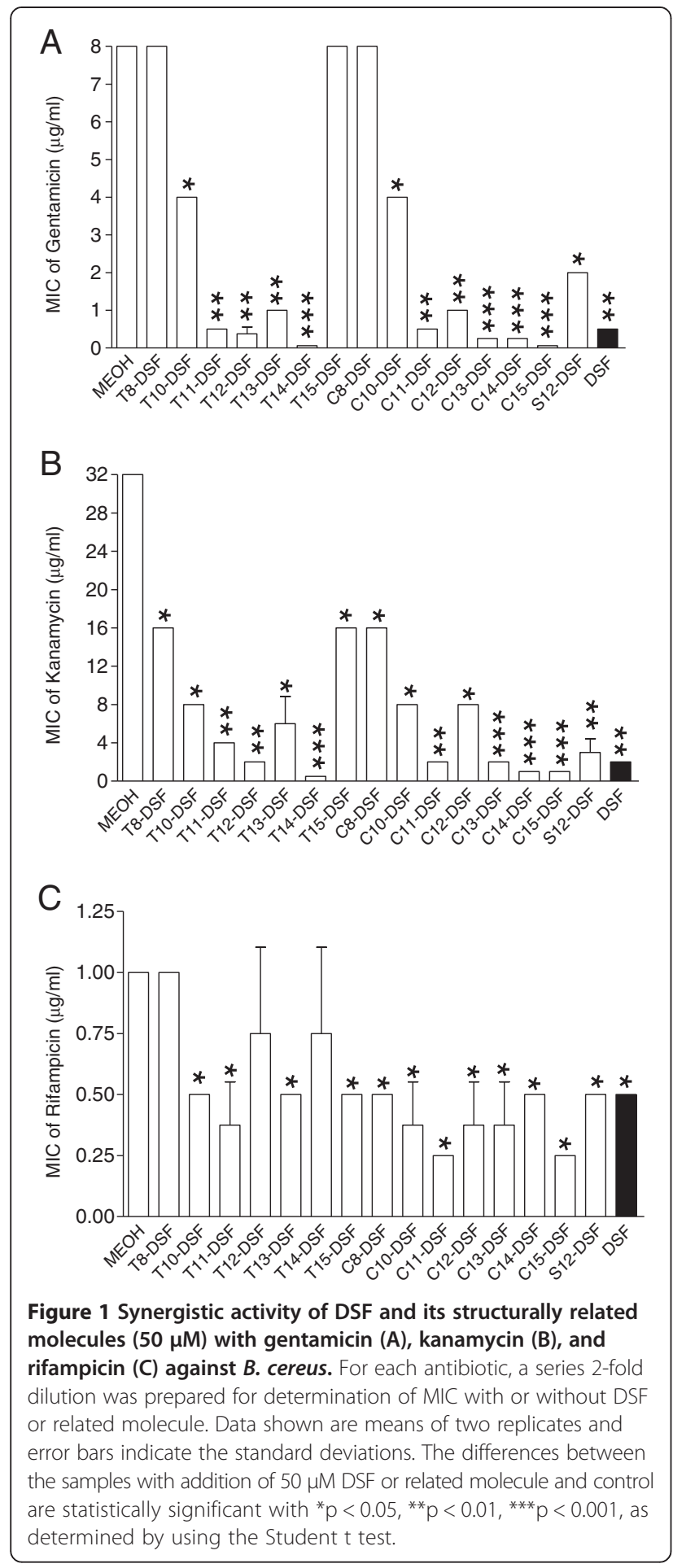

growth and biofilm formation. As shown in Figure 4, the growth rate of $B$. cereus was only slightly reduced with addition of $50 \mu \mathrm{M}$ DSF signal, whereas the bacterial biofilm formation was substantially inhibited. Intriguingly, we also discovered that DSF signal remarkably reduced the persistence of $B$. cereus (Figure 4C). Addition of $50 \mu \mathrm{M}$ DSF signal decreased the persistence rate of $B$.
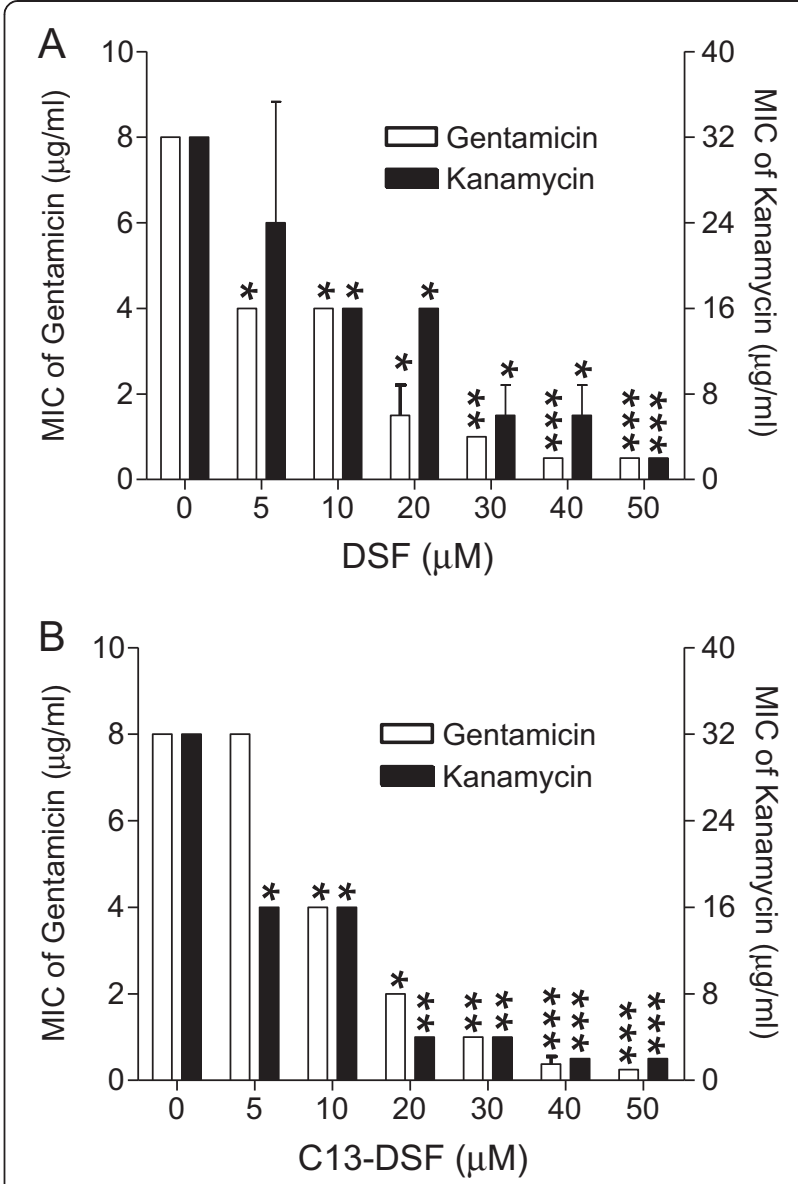

Figure 2 Synergistic activity of different concentrations of DSF (A) and C13-DSF (B) with gentamicin and kanamycin on $B$. cereus. Data shown are means of two replicates and error bars indicate the standard deviations. The differences between the samples with addition of DSF or C13-DSF and control are statistically significant with ${ }^{*} p<0.05,{ }^{* *} p<0.01,{ }^{* * *} p<0.001$, as determined by using the Student $t$ test.

cereus by 5.5- and 8.7 - fold after $4 \mathrm{~h}$ and $8 \mathrm{~h}$ incubation, respectively (Figure $4 \mathrm{C}$ ). As bacterial biofilm and persisters are highly tolerant to different types of antibiotics, inhibition of biofilm formation and persistence may likely alter bacterial antibiotic susceptibility. In combination, our results suggest that DSF signal could exert multifaceted effect on $B$. cereus, such as reducing the drug-resistant activity, inhibiting biofilm formation and attenuating bacterial persistence, which might lead to altered bacterial susceptibility to antibiotics.

\section{The combination effect of DSF signal with antibiotics on other bacterial species}

To study whether DSF could also influence the antibiotic susceptibility of other bacterial species, the signal was used to test the synergistic effect with antibiotics against a few bacterial species in our collection, including Bacillus thuringiensis, Staphylococcus aureus, 


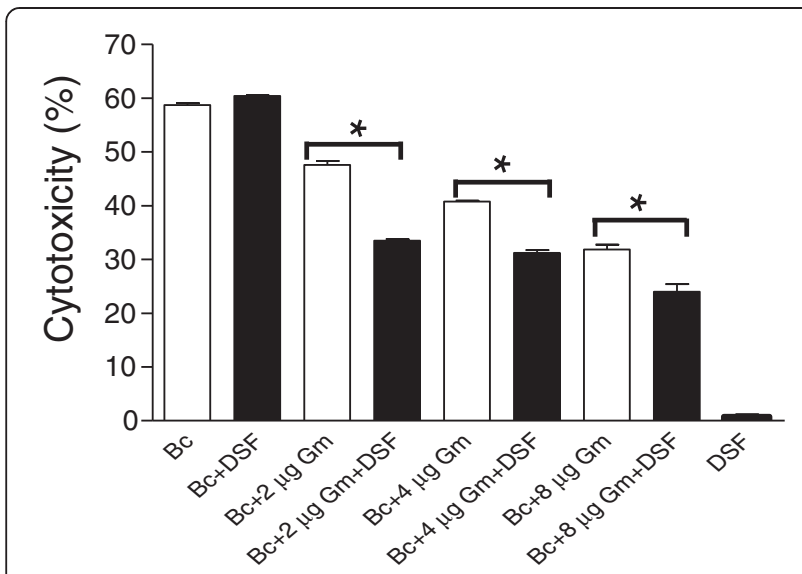

Figure 3 The synergistic effect of DSF signal $(50 \mu \mathrm{M})$ with gentamicin on the virulence of $B$. cereus in an in vitro model. Cytotoxicity was assayed by monitoring LDH release by the HeLa cells infected with a MOI of about 1000. Data shown are means of three replicates and error bars indicate the standard deviations. The differences between the samples with DSF and without DSF are statistically significant with ${ }^{*} p<0.05$, as determined by using the Student $\mathrm{t}$ test.

Mycobacterium smegmatis, Neisseria subflava, and Pseudomonas aeruginosa. Among them, B. thuringiensis belongs to $B$. cereus group and has been used as a biopesticide for many years [31]. It is closely related to the other two member of $B$. cereus group, i.e., B. anthracis and $B$. cereus, which are important human pathogens to cause anthrax and foodborne illness, respectively [32]. S. aureus is frequently found in human respiratory tract and on the skin. It can cause a range of serious illnesses such as pneumonia, meningitis, osteomyelitis, endocarditis, toxic shock syndrome (TSS), bacteremia, and sepsis [33]. M. smegmatis is a useful model organism for research analysis of other Mycobacteria species, especially $M$. tuberculosis. It is generally considered to be a non-pathogenic bacterium, however, in rare cases it may also cause diseases [34]. N. subflava is a rare opportunistic pathogen and has been associated with endocarditis, bacteremia, meningitis, septic arthritis, endophthalmitis, and septicemia [35]. P. aeruginosa is a ubiquitous environmental organism that can infect animals, plants, and insects, and is a major source of opportunistic infections in immunocompromised patients and cystic fibrosis individuals [36].

As shown in Table 2, addition of DSF signal at a final concentration of $50 \mu \mathrm{M}$ decreased the MICs of ampicillin, rifampicin, kanamycin, gentamicin, tetracycline, chloramphenicol, and trimethoprim against $B$. thuringiensis by $75 \%, 75 \%, 93.75 \%, 93.75 \%, 50 \%, 50 \%$, and $75 \%$, respectively. We then continued to test the synergistic effect of DSF signal with antibiotics against $S$. aureus. Inclusion of DSF signal at a final concentration of $50 \mu \mathrm{M}$ caused reduction of the MICs of ampicillin, kanamycin and
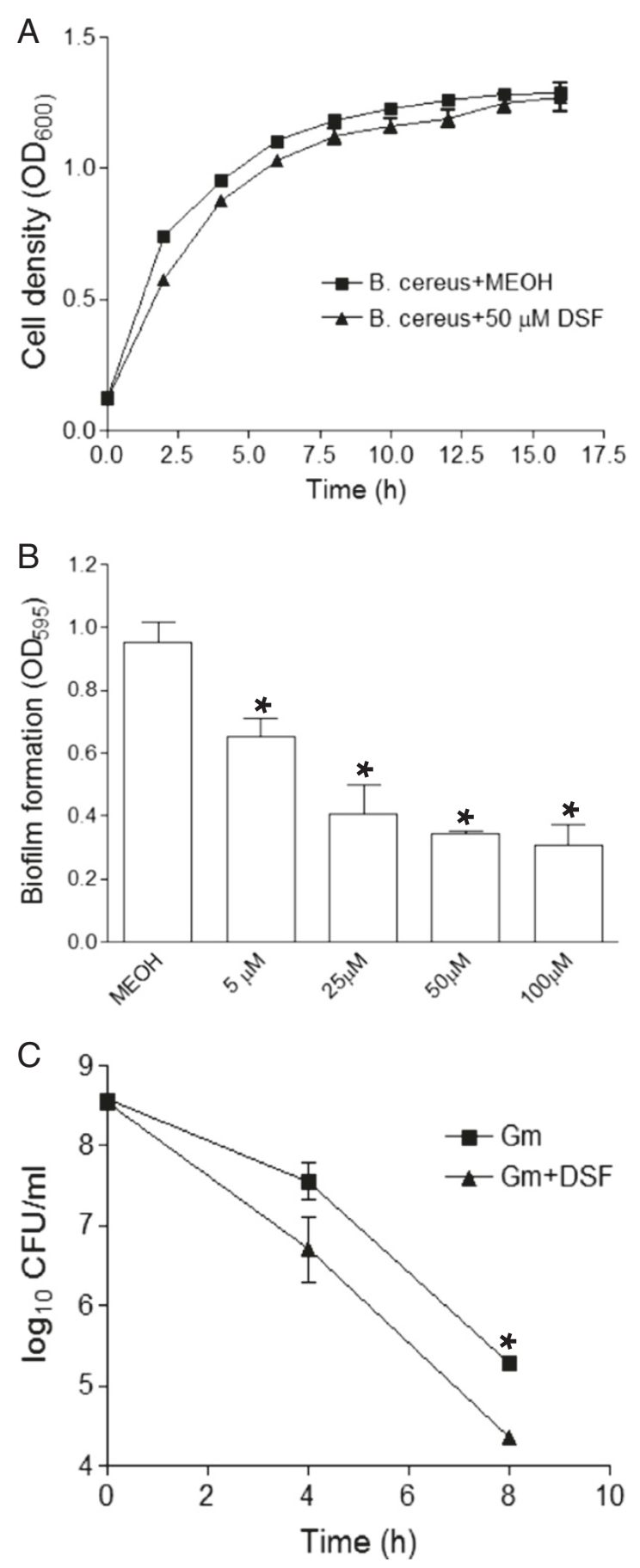

Figure 4 Influences of exogenous addition of DSF signal on the bacterial growth rate (A) biofilm formation (B), and persistence (C) of $B$. cereus. For measurement of growth rate, the bacterial cells were grown in the absence or presence of $50 \mu \mathrm{M}$ DSF; while for test of persistence, the bacterial cells were treated with $10 \mu \mathrm{g} / \mathrm{ml}$ gentamicin $(\mathrm{Gm})$ in the absence or presence of $50 \mu \mathrm{M}$ DSF signal. For biofilm formation assays, DSF signal was added at different final concentrations as indicated. Data shown are means of three replicates and error bars indicate the standard deviations. The differences between the samples with DSF and without DSF are statistically significant with ${ }^{*} p<0.05$, as determined by using the Student $t$ test. 
Table 2 Synergistic activity of DSF signal ( $50 \mu \mathrm{M})$ with antibiotics against various bacterial species

\begin{tabular}{lllllllll}
\hline & & \multicolumn{7}{c}{ MIC $(\boldsymbol{\mu g} / \mathbf{m l})$} \\
\cline { 3 - 9 } Bacteria & & $\mathbf{G m}^{*}$ & $\mathbf{K m}$ & $\mathbf{R m}$ & $\mathbf{A m}$ & $\mathrm{Tc}$ & $\mathbf{C m}$ & $\mathbf{T m}$ \\
\hline B. thuringiensis & $\mathrm{MEOH}$ & 4 & 32 & 1 & 1 & 4 & 4 & 512 \\
& $\mathrm{DSF}$ & 0.25 & 2 & 0.25 & 0.25 & 2 & 2 & 128 \\
S. aureus & $\mathrm{MEOH}$ & 0.125 & 2 & 0.0625 & 2 & 4 & 4 & NA\# \\
& $\mathrm{DSF}$ & 0.016 & 1 & 0.0625 & 1 & 4 & 4 & NA \\
M. smegmatis & $\mathrm{MEOH}$ & 0.16 & 0.32 & $\mathrm{NA}$ & 256 & 0.16 & 6.4 & 0.64 \\
& $\mathrm{DSF}$ & 0.08 & 0.08 & $\mathrm{NA}$ & 256 & 0.16 & 3.2 & 0.32 \\
N. subflava & $\mathrm{MEOH}$ & 2 & 8 & 0.5 & 2 & 2 & 0.5 & 128 \\
& $\mathrm{DSF}$ & 1 & 4 & 0.5 & 2 & 2 & 0.5 & 128 \\
P. aeruginosa & $\mathrm{MEOH}$ & 1.28 & 128 & $\mathrm{NA}$ & 128 & 32 & 128 & 64 \\
& $\mathrm{DSF}$ & 0.64 & 64 & $\mathrm{NA}$ & 128 & 32 & 128 & 64
\end{tabular}

*Abbreviations: $\mathrm{Gm}$ gentamicin, $\mathrm{Km}$ kanamycin, $\mathrm{Rm}$ rifampicin, $\mathrm{Am}$ ampicillin, Tc tetracycline, Cm chloramphenicol, and Tm trimethoprim.

\# NA means the bacterial species was not sensitive to the tested antibiotic.

gentamicin by $50 \%, 50 \%$, and $87.5 \%$, respectively (Table 2 ). While for M. smegmatis, addition of DSF signal increased its susceptibility to kanamycin, gentamicin, chloramphenicol and trimethoprim by $75 \%, 50 \%, 50 \%$ and $50 \%$, respectively (Table 2). For the synergistic effect of DSF signal with antibiotics against the Gram-negative bacterial pathogens, as shown in Table 2, it was found that addition of DSF only reduced the MICs of kanamycin and gentamicin against $N$. subflava and P. aeruginosa by $50 \%$, respectively, but did not affect the MICs of other antibiotics against these two pathogens. Furthermore, we also studied the effect of DSF-family signals on the growth rate of these bacteria, as shown in Additional file 1: Figure S2, exogenous addition of DSF-family signals showed no influence on the growth of $P$. aeruginosa, but they slightly affected the growth of B. thuringiensis, S. aureus and M. smegmatis; and inhibited the growth of $N$. subflava, which may affect its synergistic effect with antibiotics on this particular pathogen.

\section{Discussion}

Previous studies have established the significant roles of DSF-family signals in microbial ecology as well as in intraspecies signaling regulation [14,17,22-24,37]. It was reported that DSF signals could modulate various biological functions including virulence, biofilm formation, antibiotic resistance and persistence through interspecies communication [23,24,37]. Additionally, DSF-family signals were also found to play a role in inter-kingdom communication by inhibiting morphological transition of C. albicans $[14,17,22]$. The results from this study present a new role of DSF and its structurally related molecules, i.e., increasing the antibiotic susceptibility of some bacterial species (Figure 1, Table 2). Given that DSF at a final concentration of $5 \mu \mathrm{M}$, which appears to be a physiological relevant concentration [14,22], could substantially increase bacterial sensitivity to antibiotics (Figure 2A), it appears plausible that DSF-family signals may have a role in shaping local microbial ecology as they could reduce the competitive advantage of some community residents by down regulation of their antibiotic or toxin tolerance. Furthermore, our results also suggest that DSF and its structurally related molecules may be used as a new kind of antibiotic adjuvant for the treatment of infectious diseases caused by bacterial pathogens, subjecting to further evaluation of their toxicological and pharmacological properties.

DSF-family signals share a fatty acid carbon chain with variations in chain length, double-bond configuration, and side-chain [18]. Evidence is emerging that these structural features may contribute to their biological activity in intraspecies signalling and interspecies communication $[14,17,37]$. Our study showed that the synergistic activity of DSF and its structurally related molecules with antibiotics is influenced by their structural features. Each of these molecules has a distinct synergistic activity among which the disparity could be up to 128 -fold (Figure 1A). As a general rule, our results showed that the unsaturated long chain DSF related molecules have better synergistic activity with antibiotics, especially the aminoglycoside antibiotics, than the short chain and saturated molecules. Meanwhile, the synergistic activity of DSF and related molecules may also seem to be affected by the mode of action of antibiotics as the synergistic activities of DSF and related molecules with aminoglycoside antibiotics such as gentamicin and kanamycin were much better than with other types of antibiotics (Figure 1, Table 2).

It was reported that BDSF signalling system positively regulates the antibiotic resistance of B. cenocepacia [21]. The same research group also found that addition of DSF signal to $P$. aeruginosa could increase the bacterial antibiotic tolerance to polymyxins [23]. Intriguingly, our results suggest an opposite effect of DSF and its structurally related molecules by increasing the bacterial antibiotic susceptibility (Figure 1, Table 2). The contradictory results may be due to the differences in the bacterial species or strains and the antibiotics used in studies, which is evident from our results (Table 2). It should also be noted that DSF-family signals were shown to play dual roles in regulation of biofilm formation as they positively control the biofilm development in some bacterial species, and they could also disperse the biofilms of other bacterial species $[15,19,21,37]$.

Our results suggest that DSF and related molecules may influence the bacterial antibiotic susceptibility by multiple ways, including modulation of the biofilm formation, antibiotic resistant activity and bacterial persistence (Figure 4; Additional file 1: Table S1). In addition, 
we also examined the possibility of DSF and related molecules acting as biosurfactants to influence bacterial susceptibility to antibiotics by using rhamnolipid, which is a well characterized biosurfactants, as a control in MIC and growth analysis. We found that rhamnolipid could also increase the antibiotic susceptibility of $B$. cereus at the final concentration of $50 \mu \mathrm{M}$ (data not shown), but it also inhibits bacterial growth at this concentration and its toxicity on B. cereus cells was at least 5 -fold higher than DSF (Additional file 1: Figure S3), which complicates the comparison. With all considered, at this stage we could not rule out the possibility that DSF and related molecules may have biosurfactant property and this property may contribute to their synergistic effects with antibiotics. Furthermore, several lines of evidence from this study and previous reports seem to suggest that the signalling activity of DSF and its structurally related molecules may contribute to their ability in changing bacterial antibiotic susceptibility. Firstly, it was reported that BDSF signalling system positively controls the antibiotic resistance in B. cenocepacia, and addition of $50 \mu \mathrm{M}$ DSF signal increased the antibiotic resistance of $P$. aeruginosa to polymyxins [21,23], indicating that DSF-family signals are possibly widely involved in regulation of bacterial antibiotic resistance. Secondly, different from rhamnolipid which has a strong hydrophilic head group glycosyl, DSF and related molecules only have a very weak hydrophilic activity, suggesting that they could not be good surfactants. This notion appears to be supported by the different inhibitory activity of DSF and rhamnolipid on the growth of B. cereus (Additional file 1: Figure S3). Thirdly, our findings showed that addition of $50 \mu \mathrm{M}$ DSF signal showed no cytotoxicity to HeLa cells, didn't affect the B. cereus virulence (Figure 3), but could significantly change the expression patterns of many genes in B. cereus, some of which are known to be associate with antibiotics resistance or tolerance (Additional file 1: Table S1). Fourthly, the synergistic activity of DSF is antibiotic specific. While DSF and its structurally related molecules have strong synergistic effect with gentamicin and kanamycin against B. cereus, they showed a moderate effect with rifampicin, or even no synergistic effect with other antibiotics such as ampicillin, tetracycline (Data not shown), which may not be solely explainable with biosurfactant properties. Fifthly, the synergistic effect of DSF with antibiotics is also bacterial species specific. We showed that DSF signal had a strong synergistic effect with gentamicin against $B$. cereus, B. thuringiensis and $S$. aureus, while it had only a moderate effect with gentamicin against M. smegmatis, N. subflava and P. aeruginosa (Figure 1, Table 2). In particular, DSF signal did not show any synergistic activity with any of the tested antibiotics, including gentamicin, kanamycin, rifampicin, ampicillin, tetracycline, chloramphenicol, and trimethoprim, against Escherichia coli (Data not shown). Finally, DSF and its structurally related molecules share a very similarly chemical structure, hydrophobic and hydrophilic properties, suggesting that they should have similar chemical properties. However, their synergistic activities were significantly different with disparity up to 128 folds (Figure 1A).

Taken together, the results from this study have established the role of DSF and its structurally related molecules in modulation of antibiotic susceptibility in some but not all bacterial pathogens. It is also clear that the synergistic activity with antibiotics is related to the structural features of DSF-related molecules and likely the chemical property or the mode of action of antibiotics. At least stage, it is not clear how DSF and its structurally related molecules could influence bacterial antibiotic sensitivity. Much work remains to be done to determine whether their functionality in modulating bacterial antibiotic sensitivity is related to their pure chemical properties such as biosurfactant or hydrophobic activities, or associated with their potential roles in interference of bacterial signalling and regulatory networks, or both. In this regard, DSF and its analogues may be served as a useful tool to probe the potential mechanisms governing bacterial sensitivity to antibiotics.

\section{Conclusions}

In summary, we showed that DSF and its structurally related molecules could significantly increase bacterial susceptibility to antibiotics, especially gentamycin and kanamycin. Our data showed that the unsaturated long chain DSF related molecules have better synergistic activity with antibiotics, especially the aminoglycoside antibiotics, than the short chain and saturated molecules. This synergistic effect is generic on both Gram-positive and Gram-negative bacteria, but the tested Gram-positive bacteria appeared to be more sensitive to the activity of DSF and its structurally related molecules than the tested Gram-negative bacteria. The findings from this study suggest that DSF and its structurally related molecules may be used as antibiotic adjuvants, which could be useful for reducing the dose of antibiotics, hence minimizing the side effect caused by the antibiotics, and slowing down the development of antibiotic resistance.

\section{Methods}

\section{Bacterial growth conditions and MIC assays}

Bacterial strains used in this work are listed in Additional file 1: Table S2. Overnight cultures of bacteria were inoculated at an $\mathrm{OD}_{600}$ of 0.025 in LB broth supplemented with antibiotic in the absence and presence of DSF or its structural analogue (Table 1). One hundred microliters of inoculated culture were grown in each well at $28^{\circ} \mathrm{C}$ or $37^{\circ} \mathrm{C}$ as indicated with shaking at $200 \mathrm{rpm}$ for 
24 hours (Additional file 1: Table S2). MIC was defined as the lowest concentration of antibiotic in which bacterial growth in the well was not measureable by determination of the turbidity at $600 \mathrm{~nm}$, and determined following the method from the Clinical and Laboratory Standards Institute (CLSI) [38].

\section{Bacterial growth analysis}

Overnight bacterial cultures grown in LB broth were inoculated in the same medium to an $\mathrm{OD}_{600}$ of 0.025 in the absence and presence of DSF or its analogue at a final concentration of $50 \mu \mathrm{M}$. Three hundred microliters of inoculated culture were grown in each well at $28^{\circ} \mathrm{C}$ or $37^{\circ} \mathrm{C}$ as indicated in Additional file 1: Table S2 in a low intensity shaking model using the Bioscreen-C Automated Growth Curves Analysis System (OY Growth Curves AB Ltd., Finland).

\section{Biofilm formation assays}

Biofilm formation was assayed using 96-well polypropylene microtitre dishes. Overnight bacterial cultures grown in LB broth were inoculated in the same medium to an $\mathrm{OD}_{600}$ of 0.01 in the absence and presence of DSF signal at different concentrations as indicated. One hundred microliters of inoculated culture were grown in each well at $37^{\circ} \mathrm{C}$ with shaking at $150 \mathrm{rpm}$ for $18 \mathrm{~h}$. The cultures were removed and $200 \mu \mathrm{l}$ of $1 \%$ crystal violet (w/v) was added. Following staining at room temperature for $15 \mathrm{~min}$, the dye was removed and the wells were rinsed three times with water. For quantification of the attached bacterial cells, the stained wells were decolorized with $200 \mu \mathrm{l}$ of $95 \%$ ethanol. The quantity of crystal violet was determined by measuring the absorbance at $595 \mathrm{~nm}$.

\section{Persistence assays}

Persistence was measured by determining the number of $\mathrm{cfu} / \mathrm{mL}$ after exposure to $10 \mu \mathrm{g} / \mathrm{mL}$ gentamicin. Overnight cultures were diluted 100 -fold in $10 \mathrm{~mL}$ of fresh medium and incubated at $37^{\circ} \mathrm{C}$ at $250 \mathrm{rpm}$ to an $\mathrm{OD}_{600}$ of 1.0. Cultures were incubated with shaking at $150 \mathrm{rpm}$ at $37^{\circ} \mathrm{C}$ supplemented with gentamicin in the absence and presence of DSF signal at a final concentration of $50 \mu \mathrm{M}$. For determination of $\mathrm{cfu}, 1-\mathrm{mL}$ aliquots were removed at the indicated time points and cells were serially diluted in fresh medium and plated on solid medium. Persisters were calculated after incubation at $37^{\circ} \mathrm{C}$ overnight.

\section{Cytotoxicity assays in HeLa cell model}

The synergistic effect of DSF signal with antibiotic on the virulence of $B$. cereus was assayed by using HeLa cells. HeLa cells were seeded in 24-well tissue culture plates containing Dulbecco's Modified Eagle Medium (DMEM) and allowed to grow at $37^{\circ} \mathrm{C}$ in $\mathrm{CO}_{2}$ for about
18 hours to obtain $80-90 \%$ monolayer confluency $\left(5.0 \times 10^{5}\right.$ cells/well $)$. Culture supernatants were removed and the monolayer was washed once with PBS buffer. Fresh bacterial cells cultured to an $\mathrm{OD}_{600}$ of 1.0 were diluted in DMEM with or without DSF at a final concentration of $50 \mu \mathrm{M}$, which were then added to the HeLa cell monolayers at a multiplicity of infection (MOI) about 1000, and gentamycin was added at different final concentrations as indicated. Cytotoxicity was determined by measuring the release of the cytosolic enzyme lactate dehydrogenase (LDH) into supernatants using the cytotoxicity detection kit (Roche).

\section{Additional file}

Additional file 1: Figure S1. Real-time PCR analysis of DSF effect on transcriptional expression of selected genes in B. cereus 10987. Table S1. The genes with increased or decreased expression in B. cereus 10987 after treatment with 50 MM DSF. Figure S2. The bacterial growth rate in the presence and absence of $50 \mu \mathrm{M}$ DSF or its analogue. Figure S3. Effect of DSF signal and rhamnolipid on the growth rate of $B$. thuringiensis. Table S2. Bacterial strains used in this study.

\section{Competing interests}

The authors declare that they have no competing interests.

\section{Authors' contributions}

Experiments were carried out by $\mathrm{YD}, \mathrm{AL}, J \mathrm{~L}, \mathrm{SC}, \mathrm{SA}, \mathrm{YHD}$. Data analysis was finished by YD and LHZ. The study was designed by YD and LHZ, who also drafted the manuscript. All authors read and approved the final manuscript.

\section{Acknowledgements}

The funding for this work was provided by the Biomedical Research Council, the Agency of Science, Technology and Research ( $A^{*}$ Star), Singapore.

Received: 27 June 2013 Accepted: 21 February 2014

Published: 27 February 2014

\section{References}

1. Livermore DM: The need for new antibiotics. Clin Microbiol Infect 2004, 10:1-9.

2. Pfaller MA, Jones RN, Doerm GV, Kugler K: Bacterial pathogens isolated from patients with bloodstream infection: frequencies of occurrence and antimicrobial susceptibility patterns from the SENTRY antimicrobial surveillance program (United States and Canada, 1997). Antimicrob Agents Chemother 1998, 42:1762-1770.

3. Slama TG, Amin A, Brunton SA, File TM Jr, Milkovich G, Rodvold KA, Sahm DF, Varon J, Weiland D Jr: A clinician's guide to the appropriate and accurate use of antibiotics: the Council for Appropriate and Rational Antibiotic Therapy (CARAT) criteria. Am J Med 2005, 118(suppl):1-6.

4. Giannini AJ, Black HR: Psychiatric, psychogenic and somatopsychic disorders handbook. Garden City, NY: Medical Examination Publishing Co.; 1987:136-137.

5. Sundin DP, Sandoval R, Molitoris BA: Gentamicin inhibits renal protein and phospholipid metabolism in rats: implications involving intracellular trafficking. J Am Soc Nephrol 2001, 12:114-123.

6. Aaron SD, Ferris W, Henry DA, Speert DP, Macdonald NE: Multiple combination bactericidal antibiotic testing for patients with cystic fibrosis infected with Burkholderia cepacia. Am J Respir Crit Care Med 2000, 161:1206-1212.

7. Athamna A, Athamna M, Nura A, Shlyakov E, Bast DJ, Farrell D, Rubinstein E: Is in vitro antibiotic combination more effective than single-drug therapy against anthrax? Antimicrob Agents Chemother 2005, 49:1323-1325.

8. Ejim L, Farha MA, Falconer SB, Wildenhain J, Coombes BK, Tyers M, Brown ED, Wright GD: Combinations of antibiotics and nonantibiotic drugs enhance antimicrobial efficacy. Nat Chem Biol 2011, 7:348-350. 
9. Le T, Bayer AS: Combination antibiotic therapy for infective endocarditis. Clin Infect Dis 2003, 36:615-621.

10. Kristiansen JE, Hendricks O, Delvin T, Butterworth TS, Aagaard L, Christensen JB, Flores VC, Keyzer H: Reversal of resistance in microorganisms by help of non-antibiotics. J Antimicrob Chemother 2007, 59:1271-1279.

11. Lehtinen J, Lilius EM: Promethazine renders Escherichia coli susceptible to penicillin G: real-time measurement of bacterial susceptibility by fluoro-luminometry. Int J Antimicrob Agents 2007, 30:44-51.

12. Mazumdar K, Dastidar SG, Park JH, Dutta NK: The anti-inflammatory non-antibiotic helper compound diclofenac: an antibacterial drug target. Eur J Clin Microbiol Infect Dis 2009, 28:881-891.

13. Barber CE, Tang JL, Feng JX, Pan MQ, Wilson TJ, Slater H, Dow JM, Williams P, Daniels MJ: A novel regulatory system required for pathogenicity of Xanthomonas campestris is mediated by a small diffusible signal molecule. Mol Microbiol 1997, 24:555-566.

14. Wang LH, He Y, Gao Y, Wu JE, Dong YH, He C, Wang SX, Weng LX, Xu JL, Tay L, Fang RX, Zhang LH: A bacterial cell-cell communication signal with cross-kingdom structural analogues. Mol Microbiol 2004, 51:903-912.

15. He YW, Zhang LH: Quorum sensing and virulence regulation in Xanthomonas campestris. FEMS Microbiol Rev 2008, 32:842-857.

16. Deng $Y$, Boon C, Eberl L, Zhang LH: Differential modulation of Burkholderia cenocepacia virulence and energy metabolism by quorum sensing signal BDSF and its synthase. J Bacterio/ 2009, 191:7270-7278.

17. Deng Y, Wu J, Eberl L, Zhang LH: Structural and functional characterization of diffusible signal factor family quorum-sensing signals produced by members of the Burkholderia cepacia complex. Appl Environ Microbiol 2010, 76:4675-4683.

18. Deng Y, Wu JE, Tao F, Zhang LH: Listening to a new language: DSF-based quorum sensing in Gram-negative bacteria. Chem Rev 2011, 111:160-173.

19. Deng Y, Schmid N, Wang C, Wang J, Pessi G, Wu D, Lee J, Aguilar C, Ahrens CH, Chang C, Song H, Eberl L, Zhang LH: Cis-2-dodecenoic acid receptor RpfR links quorum-sensing signal perception with regulation of virulence through cyclic dimeric guanosine monophosphate. Proc Natl Acad Sci U S A 2012, 109:15479-15484.

20. Schmid N, Pessi G, Deng Y, Aguilar C, Carlier AL, Grunau A, Omasits U, Zhang LH, Ahrens CH, Eberl L: The AHL- and BDSF-dependent quorum sensing systems control specific and overlapping sets of genes in Burkholderia cenocepacia H111. PLoS One 2012, 7(11):e49966.

21. McCarthy $Y$, Yang L, Twomey KB, Sass A, Tolker-Nielsen T, Mahenthiralingam E, Dow JM, Ryan RP: A sensor kinase recognizing the cell-cell signal BDSF (cis-2-dodecenoic acid) regulates virulence in Burkholderia cenocepacia. Mol Microbiol 2010, 77:1220-1236.

22. Boon C, Deng Y, Wang LH, He Y, Xu JL, Fan Y, Pan SQ, Zhang LH: A novel DSF-like signal from Burkholderia cenocepacia interferes with Candida albicans morphological transition. ISME J 2008, 2:27-36.

23. Ryan RP, Fouhy Y, Garcia BF, Watt SA, Niehaus K, Yang L, Tolker-Nielsen T, Dow JM: Interspecies signalling via the Stenotrophomonas maltophilia diffusible signal factor influences biofilm formation and polymyxin tolerance in Pseudomonas aeruginosa. Mol Microbiol 2008, 68:75-86.

24. Twomey KB, O'Connell OJ, McCarthy Y, Dow JM, O'Toole GA, Plant BJ, Ryan RP: Bacterial cis-2-unsaturated fatty acids found in the cystic fibrosis airway modulate virulence and persistence of Pseudomonas aeruginosa. ISMEJ 2012, 6:939-950.

25. Davies DG, Marques CNH: A fatty acid messenger is responsible for inducing dispersion in microbial biofilm. J Bacterio/ 2009, 191:1393-1403.

26. Costerton JW, Lewandowski Z, Caldwell DE, Korber DR, Lappin-Scott HM: Microbial biofilms. Annu Rev Microbiol 1995, 49:711-745.

27. Moir A, Corfe BM, Behravan J: Spore germination. Cell Mol Life Sci 2002, 59:403-409.

28. Driks A: Maximum shields: the armor plating of the bacterial spore. Trends Microbiol 2002, 10:251-254

29. Turnbull PC: Introduction: anthrax history, disease, and ecology. Curr Top Microbiol Immunol 2002, 271:1-19.

30. Kotiranta A, Lounatmaa K, Haapasalo M: Epidemiology and pathogenesis of Bacillus cereus infections. Microbes Infect 2000, 2:189-198.

31. Addison JA: Persistence and nontarget effects of Bacillus thuringiensis in soil: a review. Can J Forensic Res 1993, 23:2329-2342.

32. Helgason E, Okstad OA, Caugant DA, Johansen HA, Fouet A, Mock M, Hegna I, Kolstø AB: Bacillus anthracis, Bacillus cereus, and Bacillus thuringiensis-one species on the basis of genetic evidence. Appl Environ Microbiol 2000, 66:2627-2630
33. Kluytmans J, Belkum AV, Verbrugh H: Nasal carriage of Staphylococcus aureus: epidemiology, underlying mechanisms, and associated risks. Clin Microbiol Rev 1997, 10:505-520.

34. Collins FM: Mycobacterial disease, immunosuppression, and acquired immunodeficiency syndrome. Clin Microbiol Rev 1989, 2:360-377.

35. Pollack S, Mogtader A, Lange M: Neisseria subflava endocarditis. Case report and review of the literature. Am J Med 1984, 76:752-758.

36. Bodey GP, Bolivar R, Fainstein V, Jadeja L: Infections caused by Pseudomonas aeruginosa. Rev Infect Dis 1983, 5:279-313.

37. Deng Y, Boon C, Chen S, Lim A, Zhang LH: Cis-2-dodecenoic acid signal modulates virulence of Pseudomonas aeruginosa through interference with quorum sensing systems and T3SS. BMC Microb 2013, 13:231.

38. Andrews JM: Determination of minimum inhibitory concentrations. J Antimicrob Chemother 2001, 48(Suppl 1):5-16.

doi:10.1186/1471-2180-14-51

Cite this article as: Deng et al:: Diffusible signal factor (DSF) quorum sensing signal and structurally related molecules enhance the antimicrobial efficacy of antibiotics against some bacterial pathogens. BMC Microbiology 2014 14:51

\section{Submit your next manuscript to BioMed Central and take full advantage of:}

- Convenient online submission

- Thorough peer review

- No space constraints or color figure charges

- Immediate publication on acceptance

- Inclusion in PubMed, CAS, Scopus and Google Scholar

- Research which is freely available for redistribution 